\title{
Data-Centric Intelligent Computing
}

\author{
Jun Shen ${ }^{1}$, Chih-Cheng Hung ${ }^{2}$, Ghassan Beydoun ${ }^{3}, \mathrm{Yan} \mathrm{Li}^{4}$, William Guo ${ }^{5}$ \\ ${ }^{I}$ School of Computing and Information Technology, University of Wollongong \\ Northfields Avenue, Wollongong, NSW 2522 Australia \\ Email: jshen@uow.edu.au \\ ${ }^{2}$ College of Computing and Software Engineering, Kennesaw State University \\ 1100 South Marietta Pkwy, Marietta, GA 30060,USA Email: chungl@kennesaw.edu \\ ${ }^{3}$ Faculty of Engineering and Information Technology, University of Technology Sydney \\ PO Box 123, Broadway, NSW 2007, Australia \\ Email: Ghassan.Beydoun@uts.edu.au \\ ${ }^{4}$ School of Agricultural, Computational and Environmental Sciences, University of Southern Queensland \\ USQ Toowoomba Campus, West Street, Toowoomba QLD 4350, Australia \\ Email:Yan.Li@usq.edu.au \\ ${ }^{5}$ School of Engineering and Technology, Central Queensland University \\ Bruce Highway, North Rockhampton QLD 4702, Australia \\ Email:w.guo@cqu.edu.au
}

\section{Preface}

In the age of Big Data and Internet of Things the integration of traditional and contemporary intelligent computing techniques continues to play an increasingly vital role in data analysis, real-time control and operation, decision making, and system evaluation and forecasting. Many computational intelligence methods and techniques, such as fuzzy logic and genetic algorithms, were initially proposed to deal with a certain type of datasets, whose success then led to the generalization of many algorithms that can deal with common types of datasets.

With the rapid advancement in pervasive computing and integration with big datasets, such digital datasets in different formats have been exponentially collected from different organisations and projects. People and organisations often need to deal with these heterogeneous datasets nowadays; hence expect integrated data-centric computing algorithms and/or systems to meet the needs of their business activities.
Therefore, integrated data-centric intelligent computing and systems are finding increasingly more applications from community-based business transactions to intelligent transport systems.

This themed special issue is to facilitate the exchange and dissemination of novel algorithms, system design and development, and advanced applications in integrated data-centric intelligent computing and system development in all disciplines. This special issue focuses on applications of intelligent computing approaches to solve increasingly complex problems.

By filtering solicited abstracts, we received 24 full paper submissions and 18 of them entered review process. Every paper was evaluated by 2-3 external experts in its relevant field, and was requested to revise deeply. After two rounds of vigorous peer review process for each submission, the guest editors and editors-in-chief are pleased to accept 5 papers, which technical quality and relevance of scope are satisfactory to the journal. 
The paper 'Multi-feature based Event Recommendation in Event-Based Social Network', co-authored by Jiuxin Cao, Ziqing Zhu, Liang Shi, Bo Liu and Zhuo Ma, integrated different features in event based social network to propose novel algorithms, namely MHF and MHF-L, for the design of recommendation system. Similar for designing an online evolutionary recommendation system, Yongheng Chen, Chunyan Yin, Yaojin Lin and Wan Li Zuo's paper 'On-line Evolutionary Sentiment Topic Analysis Modeling' focused on sentiment topic analysis and modelling and designed a new algorithm, namely OESTM, which significantly improved traditional non-parametric Bayes methods.

On the other hand, in the paper 'A Comparison of Outlier Detection Techniques for High-Dimensional Data', Xiaodan Xu, Huawen Liu, Li Li and Minghai Yao provided a decent and comprehensive review on the challenging research topic related to outlier detection, but with refreshing insights for complex data centric applications.
The other two papers in this special issue have dealt with more specific data centric intelligent computing applications. Paper 'A Computer Aided Analysis Scheme for Detecting Epileptic Seizure from EEG Data', co-authored by Enamul Kavir, Siuly and Hua Wang, investigated machine learning methods for detecting epileptic seizure from electroencephalogram, which is very important in biomedical informatics fields. In the paper 'Data-Intensive Service Provision Based on Particle Swarm Optimization', Lijuan Wang and Jun Shen were more concerned with how to apply PSO in services and cloud computing contexts.

We do hope the selection of the accepted papers will contribute to the themed research area. And also, we deeply and gratefully appreciate the timely, careful and constructive evaluations from invited reviewers, and also the patience and support from the journal's editorial and managerial team to make this special issue successfully published. 